\title{
Microarray analysis identifies defects in regenerative and immune response pathways in COPD airway basal cells
}

\author{
Fanny Pineau ${ }^{1}$, Gabriella Shumyatsky ${ }^{1}$, Nicole Owuor ${ }^{1}$, Nisha Nalamala ${ }^{1}$, \\ Sudhir Kotnala ${ }^{1}$, Sudhir Bolla ${ }^{1}$, Nathaniel Marchetti ${ }^{1}$, Steven Kelsen ${ }^{1}$, \\ Gerard J. Criner ${ }^{1}$ and Uma S. Sajjan (1) ${ }^{1,2}$
}

Affiliations: 'Dept of Thoracic Surgery and Medicine, Temple University, Philadelphia, PA, USA. ${ }^{2}$ Dept of Physiology, Lewis Katz Medical School, Temple University, Philadelphia, PA, USA.

Correspondence: Uma S. Sajjan, Temple University, 3500 N. Broad Street, Philadelphia, PA 19140, USA.

E-mail: uma.sajjandatemple.edu

\section{ABSTRACT}

Background: Airway basal cells are specialised stem cells and regenerate airway epithelium. Airway basal cells isolated from patients with COPD regenerate airway epithelium with an abnormal phenotype. We performed gene expression analysis to gain insights into the defective regenerative programme in COPD basal cells.

Methods: We conducted microarray analysis and compared COPD versus normal basal cells to identify differentially regulated genes (DEGs) and the enriched biological pathways. We determined the correlation of DEGs with cell polarisation and markers of ciliated and goblet cells. HOXB2 was knocked down in $16 \mathrm{HBE} 14 \mathrm{o}-$ cells and monitored for polarisation of cells. HOXB2 expression in the lung sections was determined by immunofluorescence.

Results: Comparison of normal and COPD basal cell transcriptomic profiles highlighted downregulation of genes associated with tissue development, epithelial cell differentiation and antimicrobial humoral response. Expression of one of the tissue development genes, HOXB2 showed strong correlation with transepithelial resistance and this gene was downregulated in COPD basal cells. Knockdown of HOXB2, abrogated polarisation of epithelial cells in normal cells. Finally, HOXB2 expression was substantially reduced in the bronchial epithelium of COPD patients.

Conclusions: Defect in gene signatures involved in tissue development and epithelial differentiation were implicated in COPD basal cells. One of the tissue developmental genes, HOXB2, is substantially reduced in bronchial epithelium of COPD patients. Since HOXB2 contributes to airway epithelial cell polarisation, we speculate that reduced expression of HOXB2 in COPD may contribute to abnormal airway epithelial regeneration in COPD.

@ERSpublications

COPD airway basal cells show downregulation of gene sets that are involved in intercellular junctions, epithelial differentiation and immune responses, highlighting the possible mechanisms of defective airway epithelial repair in COPD https://bit.ly/3kneloj

Cite this article as: Pineau F, Shumyatsky G, Owuor N, et al. Microarray analysis identifies defects in regenerative and immune response pathways in COPD airway basal cells. ERJ Open Res 2020; 6: 00656-2020 [https://doi.org/10.1183/23120541.00656-2020].

This article has supplementary material available from openres.ersjournals.com

Received: 9 Sept 2020 | Accepted: 13 Sept 2020

Copyright $\odot$ ERS 2020. This article is open access and distributed under the terms of the Creative Commons Attribution Non-Commercial Licence 4.0. 


\section{Introduction}

Epithelium lining the conductive airways protects the lungs from environmental factors. In the presence of chronic damage due to persistent inflammation and infection, the protective mechanisms of airway epithelium can malfunction, and this can contribute to the pathology of chronic lung diseases, including COPD. A significant component of dysfunction in these chronic airway diseases is associated with impaired epithelial repair leading to remodelled airway epithelium, inflammation and fibrosis [1-3].

COPD is a progressive lung disorder characterised by airflow obstruction. Emphysema, inflammation of large and small airways, mucociliary dysfunction and airway remodelling are common features of COPD $[4,5]$. Bronchial epithelium in COPD patients often shows basal cell hyperplasia, squamous metaplasia and goblet cell metaplasia.

The basal cells are specialised stem cells present in the tracheobronchial tree and regenerate the conductive airway epithelium following injury. Previously, we and others have demonstrated that basal cells isolated from COPD patients regenerate structurally altered airway epithelium with basal cell hyperplasia and goblet cell metaplasia [6-9]. Furthermore, regenerated airway epithelium from COPD basal cells show pro-inflammatory phenotype and reduction in barrier function $[10,11]$. Now there is compelling evidence that basal cells play a pivotal role in the early pathogenesis of COPD [9, 12-14]. Therefore, it is necessary to understand the mechanisms of regenerative pathways that are defective in COPD basal cells.

Comparison of transcriptomic profiles of airway epithelial cells from healthy nonsmokers and healthy smokers (with normal lung function and chest imaging) identified 676 differentially expressed genes (DEGs), primarily upregulated in smokers and related to development, metabolism, signal transduction, transcription and transport [15], with an enrichment of DEGs in the chromosomal sub-band 19q13.2, a COPD risk locus [16]. Paradoxically, NOTCH and WNT signalling pathways, which were demonstrated to promote goblet cell metaplasia and an inflammatory phenotype, do not appear to play a role in COPD, because these genes are downregulated in the airways of these patients $[8,17-20]$. Transcriptome analysis of airway epithelial cells isolated from bronchioles showed loss of regional transcriptome identity leading to proximalisation in smokers with COPD [21]. Further, the authors demonstrated that these changes were related to cigarette smoking. However, these findings do not explain the mechanisms underlying the goblet or basal cell hyperplasia observed in the large airways of COPD patients.

In an attempt to gain insight into what influences abnormal differentiation of COPD basal cells, we compared transcriptomic profiles of basal cells obtained from bronchial tissue of healthy never-smokers and COPD subjects (who had stopped smoking for at least 1 year at the time of tissue collection). We also cultured the basal cells at the air-liquid interface (ALI) to determine whether DEGs in basal cells correlate with polarisation and differentiation markers. We also examined the functional role of one of the DEGs, categorised under tissue development for its functional role in airway epithelial regeneration.

\section{Materials and methods}

Airway epithelial cells

Basal cells were isolated from bronchial segments of normal donor lungs and explanted lungs from COPD patients at the time of lung transplantation as described previously [7, 8]. Collection of the tissue was approved by Institutional Review Board of University Michigan, Ann Arbor, MI and Temple University, Philadelphia. Patient characteristics are provided in supplementary table 1 . The nerve growth factor receptor positive $\left(\mathrm{NGFR}^{+}\right.$) basal cells were cultured in $12 \mathrm{~mm}$ Transwells, as described previously [8, 22] and harvested either when the basal cells were $80 \%$ confluent or after 1, 2 or 4 weeks of culturing at the ALI.

\section{Microarray and Gene Ontology}

Biotinylated cDNAs synthesised from total RNA were subjected to microarray analysis using Human Gene 2.1 ST arrays at $48^{\circ} \mathrm{C}$. The linear models for microarray data methodology was applied to the $\log _{2}$-transformed expression data to identify statistically significant DEG (p-value of $<0.05$ and up/ downregulated by more than two-fold) for each comparison and further analysed. Normalised data and raw data are available in Gene Expression Omnibus with accession number GSE137557.

Gene Ontology (GO) and KEGG pathways were analysed with WebGestalt (WEB-based GEne SeT AnaLysis Toolkit) [23] using the Benjamini-Hochberg correction for multiple testing (false discovery rate of 5\%). For GO, only Biological Process terms are discussed because Cellular Component and Molecular Function terms were less relevant.

\section{Flow cytometry}

Mucociliary-differentiated cells were dissociated with accutase (Thermo Fisher Scientific, Waltham. MA, USA), cells were permeabilised with $4 \%$ paraformaldehyde, and incubated in PBS containing $1 \%$ BSA and 
0.5\% saponin. Cells were then incubated with acetylated tubulin (Sigma Aldrich, St. louis, MO, USA), Muc5AC (Abcam, Cambridge, MA, USA) or TP63 (Abcam), bound antibodies were detected by using AlexaFluor-labelled second antibodies. The cells were then analysed in FACSCalibur Flow cytometer (BD Biosciences, San Jose, CA, USA), and data were analysed by FlowJO version 10 (Tree Star, Ashland, OR, USA).

\section{Transepithelial resistance and histology}

Transepithelial resistance (TER) of airway epithelial cell cultures cultured at the ALI for 2 weeks was determined by using an EVOM volt/Ohm meter with EndOhm chambers (World Precision Instruments, Sarasota, FL, USA). For histological evaluation, cell cultures were fixed in buffered formalin, embedded in paraffin and 5- $\mu \mathrm{m}$ thick sections were deparaffinised and stained with haematoxylin and eosin $(\mathrm{H} \& \mathrm{E})$ or periodic acid Schiff (PAS) reagent.

\section{Real-time PCR}

cDNA was synthesised from total RNA and subjected to quantitative PCR (qPCR) to determine the expression of ADAMTSL3, ELF5, HOXA1, HOXB2, IVL, KRTDAP, STC2, ELF5 and VGLL1 using gene-specific primers and probes. The expression levels of each gene are presented as fold change over the housekeeping gene, glyceraldehyde 3-phosphate dehydrogenase (G3PDH).

\section{Transfection of 16HBE140- cells}

The 16HBE14o- cells (immortalised bronchial epithelial cell line) were transfected with smart pool HOXB2 short interfering RNA (siRNA) or nontargeting siRNA (Horizon Discovery, Lafayette, CO, USA), as described previously [24]. TER was measured $48 \mathrm{~h}$ after transfection to assess polarisation of cells.

\section{Immunofluorescence staining}

Cells growing in Transwells were fixed, permeabilised, blocked and incubated with antibody to E-cadherin or occludin. The bound antibodies were detected with appropriate Alexa Fluor-labelled antibodies, counter stained with 4',6-diamidino-2-phenylindole (DAPI) and imaged using a Zeiss confocal microscope [25]. Lung tissue at the second and third branching of bronchi was collected from six COPD and six healthy nonsmokers under the approval of the Temple Institute Review Board. The tissues were fixed in $10 \%$ buffered formalin, embedded in paraffin and $5-\mu \mathrm{m}$ thick sections were used for detection of HOXB2 by immunofluorescence microscopy using antibody from Developmental Studies Hybridoma Bank, University of Iowa, Iowa City, IA and using the tyramide signal amplification method.

\section{Western blot analysis}

Total protein from cells was subjected to Western blot analysis with antibodies to E-cadherin, occludin, HOXB2 and $\beta$-actin. Density of the protein bands was determined by ImageJ and expressed as fold increase over $\beta$-actin.

\section{Statistical analysis}

Data are presented as median with range. Statistical significance was assessed by nonparametric analysis, Mann-Whitney U-test to compare two groups and ANOVA on ranks with Kruskal-Wallace nonparametric test to compare three groups. A p-value $\leqslant 0.05$ was considered statistically significant.

\section{Results}

Phenotypic characterisation of airway epithelium regenerated from basal cells isolated from normal and COPD subjects

As previously observed basal cells from both normal and COPD subjects regenerated mucociliary-differentiated airway epithelium (figure 1a), with COPD cell cultures showing more goblet cells as assessed by PAS staining (figure 1b) [7, 8]. Quantification of cell types by flow cytometry showed significantly more goblet cells and basal cells and less ciliated cells in COPD than in normal samples (figure 1c). Normal cultures polarise by 2 weeks of culturing at the ALI, and polarisation of cells is necessary for the differentiation of cells. Therefore, we determined the TER at 2 weeks of culturing at the ALI, and COPD cell cultures showed significantly lower TER than normal cultures (figure 1d). To identify the possible biological pathways that lead to defective airway epithelial regeneration, we conducted transcriptome analysis on COPD and normal basal and differentiated airway epithelial cell cultures.

\section{Differentially expressed genes between COPD and normal basal cells}

To determine a COPD-specific transcriptomic signature, we compared basal cells from COPD (C) and healthy subjects $(\mathrm{N})$. In this analysis, 99 probes showed differential expression (30 upregulated, 69 downregulated in COPD), and 70 of them mapped to known transcripts (figure $2 \mathrm{a}$ and supplementary table 2). The most DEGs included HOXA1, HIST3H2BB, TENM2, SLC2A12, VGLL1, ARHGAP40 and 
a)

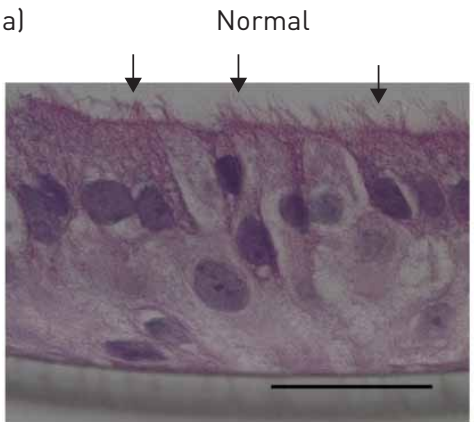

b)

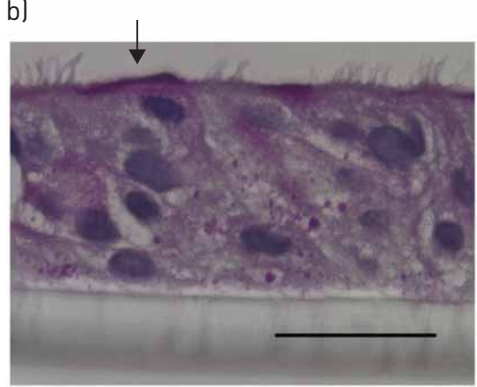

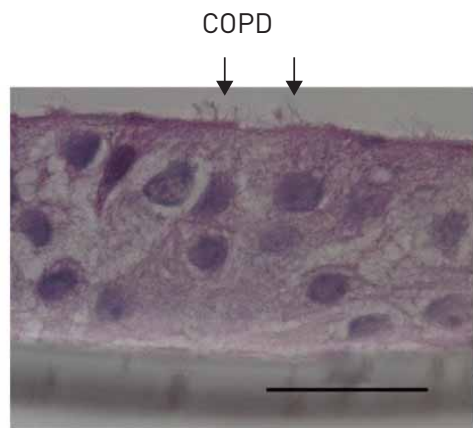

$H \& E$

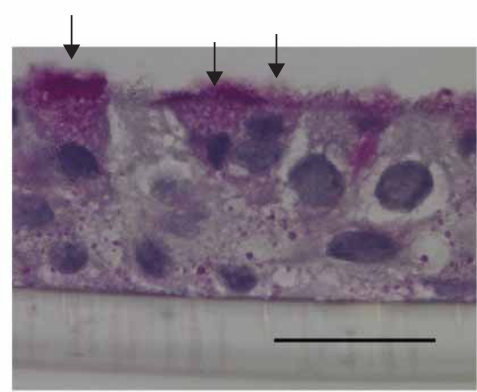

c)

\begin{tabular}{lccc}
\hline $\begin{array}{l}\text { Regenerated } \\
\text { airway epithelium }\end{array}$ & TP63 $^{+}$basal cells & $\begin{array}{c}\text { MUC5AC } \text { goblet }^{+} \\
\text {cells }\end{array}$ & $\begin{array}{c}\text { Acetylated tubulin } \\
\text { ciliated cells }\end{array}$ \\
\hline Normal & $24 \pm 3.5$ & $16 \pm 4.1$ & $41 \pm 2.5$ \\
COPD & $32 \pm 2.2^{*}$ & $25 \pm 3.6^{*}$ & $30 \pm 2.4^{*}$ \\
\hline
\end{tabular}

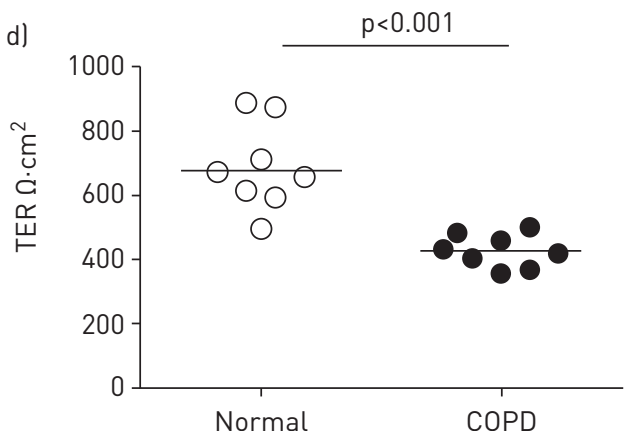

FIGURE 1 Airway epithelium regenerated from COPD basal cells shows an abnormal phenotype. Basal cells from 8 normal and 8 COPD subjects were cultured in Transwells at air-liquid interface (ALI) for 4 weeks. The Transwells were transferred to new receiver plates containing fresh medium and incubated for $24 \mathrm{~h}$ prior to harvesting. The cultures in the Transwells were fixed in 10\% buffered formalin, embedded in paraffin, and the paraffin sections were stained with a) haematoxylin and eosin (H \& E) or b) periodic acid Schiff (PAS). Arrows in panel (a) indicate cilia and arrows in panel (b) indicate goblet cells. Images in (a and b) are representative of eight COPD and eight normal regenerated airway epithelial cell cultures. c) From identical cultures, cells were dissociated and subjected to flow cytometry to determine different cell population and data represent mean \pm SD. d) Transepithelial resistance (TER) was measured after culturing basal cells at ALI for 2 weeks. Data represent median and range. The statistical significance was either determined by unpaired t-test (c) or by Mann-Whitney nonparametric test (d). Scale bars $=20 \mu \mathrm{m}$.

HOXB2 (table 1). GO analysis of the DEGs showed an overrepresentation among genes associated with epithelial/epidermal cell differentiation, cornification, and antimicrobial humoral response (supplementary table 3). All genes in these GO categories were downregulated in COPD cells. Genes involved in epithelial/ epidermal cell differentiation and cornification, and tissue development categories included genes encoding kallikreins, keratins, S100 calcium-binding protein A7 (S100A7), desmoglein-1 (DSG1), uroplakin 1A (UPK1A), E74-like factor 5 (ELF5), HOXB2, VGLL1 and KRTDAP (table 1). 


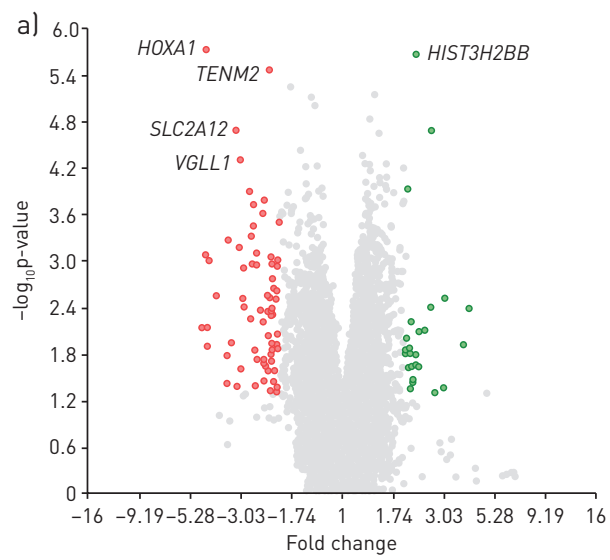

b)
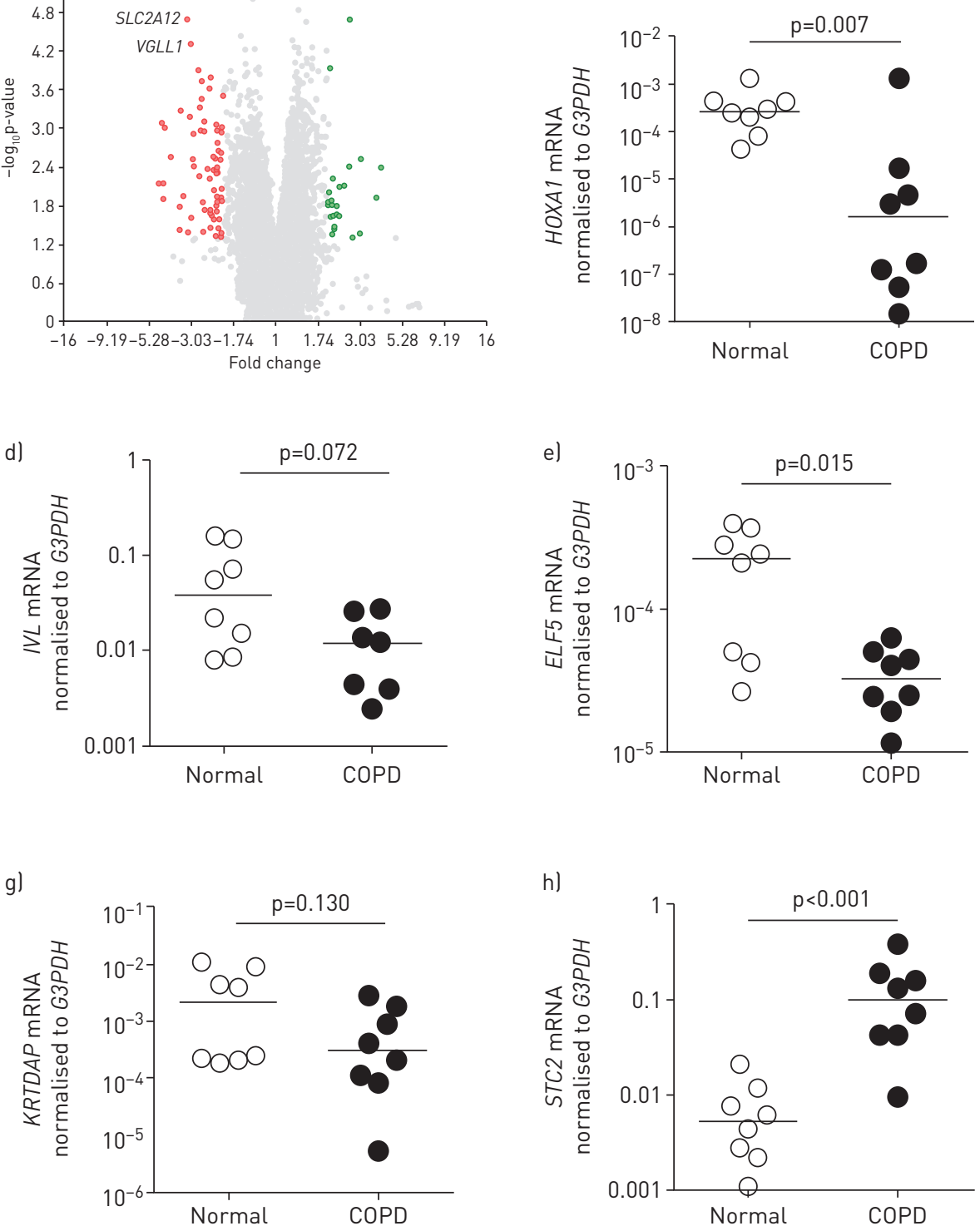

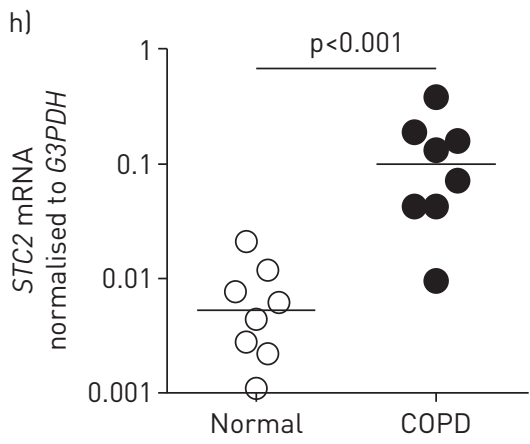

c)

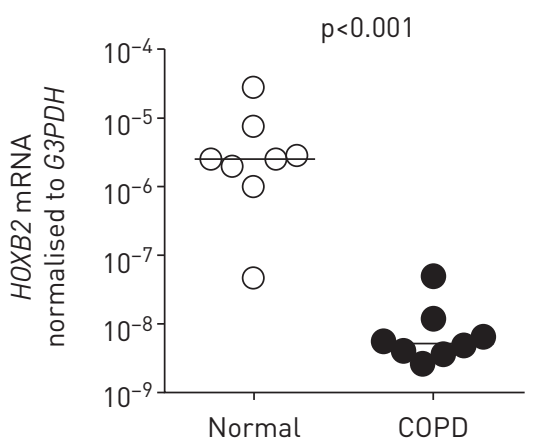

FIGURE 2 Differentially regulated genes in basal COPD and normal cells. a) Volcano plot showing differential gene expression between basal cells from 8 COPD and 8 normal subjects: red, downregulated genes; green, upregulated genes. Total RNA isolated from the basal cells was subjected to real-time quantitative PCR for b) HOXA1, c) HOXB2, d) IVL, e) ELF5, f) VGLL1, g) KRTDAP, h) STC2 and i) ADAMTSL3. Data represent median with range from 8 COPD and 8 normal subjects and statistical significance was determined by Mann-Whitney U-test.

Validation of DEGs between COPD and normal basal cells using qRT-PCR

To confirm the differences observed between COPD and normal basal cells in microarray analysis, we measured the expression levels of some of the DEGs involved in either epithelial differentiation or tissue development, and STC2 and ADAMTSL3, which are not involved in either of these categories using probe-based qPCR. HOXA1, HOXB2, STC2, ELF5 and VGLL1 showed significant differences between normal and COPD; ADAMTSL3, KRTDAP and IVL showed changes in the same direction as observed with microarray although differences were not significant (figure $2 \mathrm{~b}-\mathrm{i}$ ).

\section{Correlation of tissue development and epithelial differentiation with phenotypic features of differentiated cultures}

Next, we evaluated whether DEGs associated with tissue development/epithelial differentiation categories correlate with polarisation or differentiation. The cells cultured at the ALI from the same batch of basal cells used in the microarray analysis were used for this analysis. TER measured at 2 weeks of culturing at the ALI, and mRNA expression of FOXJ1 (marker of ciliated cells) and MUC5AC (marker of goblet cells) 


\begin{tabular}{|c|c|c|c|c|}
\hline Gene symbol & COPD & Normal & Fold change & p-value \\
\hline \multicolumn{5}{|c|}{ Top-20 downregulated genes in COPD } \\
\hline HOXA1 ${ }^{\#}$ & $1.51 \pm 0.70$ & $3.67 \pm 0.50$ & -4.47 & $1.8 \times 10^{-6}$ \\
\hline TENM2 & $6.98 \pm 0.45$ & $8.13 \pm 0.28$ & -2.21 & $3.3 \times 10^{-6}$ \\
\hline SLC2A12 & $2.93 \pm 0.57$ & $4.61 \pm 0.65$ & -3.19 & $2.0 \times 10^{-5}$ \\
\hline VGLL1" & $5.06 \pm 0.61$ & $6.67 \pm 0.57$ & -3.05 & $4.7 \times 10^{-5}$ \\
\hline ARHGAP 40 & $1.75 \pm 0.51$ & $3.21 \pm 0.72$ & -2.76 & $1.0 \times 10^{-4}$ \\
\hline CLIC3 & $5.23 \pm 0.59$ & $6.48 \pm 0.66$ & -2.38 & $2.0 \times 10^{-4}$ \\
\hline HOXB2 $2^{\#}$ & $1.89 \pm 0.31$ & $3.29 \pm 0.93$ & -2.64 & $2.0 \times 10^{-4}$ \\
\hline IL36RN & $2.76 \pm 0.45$ & $3.76 \pm 0.69$ & -2.00 & $3.0 \times 10^{-4}$ \\
\hline PADI1 & $3.31 \pm 0.63$ & $4.76 \pm 0.76$ & -2.73 & $5.0 \times 10^{-4}$ \\
\hline MFAP5 & $4.49 \pm 0.68$ & $6.31 \pm 0.85$ & -3.53 & $5.0 \times 10^{-4}$ \\
\hline ATP12A & $5.06 \pm 1.26$ & $7.23 \pm 1.40$ & -4.48 & $8.0 \times 10^{-4}$ \\
\hline FAM46C & $2.21 \pm 0.40$ & $3.23 \pm 0.60$ & -2.02 & $9.0 \times 10^{-4}$ \\
\hline MAL & $4.20 \pm 0.73$ & $6.31 \pm 1.75$ & -4.32 & $1.0 \times 10^{-3}$ \\
\hline$K L K 5^{\emptyset}$ & $4.47 \pm 0.77$ & $5.90 \pm 0.84$ & -2.68 & $1.1 \times 10^{-3}$ \\
\hline HEPHL 1 & $1.25 \pm 0.31$ & $2.59 \pm 1.16$ & -2.53 & $1.1 \times 10^{-3}$ \\
\hline LGALS9B & $4.86 \pm 0.62$ & $6.43 \pm 0.79$ & -2.97 & $1.2 \times 10^{-3}$ \\
\hline$E L F 5^{9}$ & $3.84 \pm 0.57$ & $4.92 \pm 0.93$ & -2.12 & $2.2 \times 10^{-3}$ \\
\hline$K L K 7^{\Uparrow}$ & $7.42 \pm 0.50$ & $8.45 \pm 0.93$ & -2.05 & $2.4 \times 10^{-3}$ \\
\hline SCNN1G & $3.45 \pm 0.72$ & $4.65 \pm 0.60$ & -2.31 & $2.7 \times 10^{-3}$ \\
\hline UPK1A & $1.96 \pm 0.55$ & $3.94 \pm 1.93$ & -3.96 & $2.8 \times 10^{-3}$ \\
\hline$I V L^{\text {ๆ }}$ & $3.92 \pm 1.03$ & $5.1 \pm 1.1$ & -2.27 & 0.0093 \\
\hline KRTDAP\# & $2.47 \pm 0.6$ & $3.59 \pm 1.57$ & -2.17 & 0.0114 \\
\hline S100A7ף & $1.82 \pm 0.89$ & $3.17 \pm 1.18$ & -2.55 & 0.0186 \\
\hline KRT24ף & $1.66 \pm 0.94$ & $3.28 \pm 1.15$ & -3.06 & 0.0241 \\
\hline$D S G 1^{\uparrow}$ & $2.64 \pm 1.65$ & $4.32 \pm 1.96$ & -3.21 & 0.0411 \\
\hline \multicolumn{5}{|c|}{ Top-10 upregulated genes in COPD } \\
\hline HIST3H2BB & $4.92 \pm 0.41$ & $3.78 \pm 0.38$ & 2.21 & $2.1 \times 10^{-6}$ \\
\hline CLGN & $3.83 \pm 0.80$ & $2.19 \pm 0.50$ & 3.12 & $3.0 \times 10^{-4}$ \\
\hline HIST1H3J & $5.33 \pm 0.44$ & $4.08 \pm 0.58$ & 2.39 & $3.0 \times 10^{-4}$ \\
\hline HIST1H2AB & $6.02 \pm 0.61$ & $4.51 \pm 0.51$ & 2.84 & $4.0 \times 10^{-4}$ \\
\hline ANXA10 & $6.24 \pm 1.33$ & $4.24 \pm 1.16$ & 3.99 & $4.0 \times 10^{-3}$ \\
\hline HIST1H3H & $2.70 \pm 0.26$ & $1.62 \pm 0.83$ & 2.13 & $6.0 \times 10^{-3}$ \\
\hline CHAC1 & $6.73 \pm 1.32$ & $4.83 \pm 0.45$ & 3.72 & $1.2 \times 10^{-2}$ \\
\hline CCNE2 & $5.67 \pm 0.75$ & $4.62 \pm 0.29$ & 2.07 & $1.6 \times 10^{-2}$ \\
\hline GTF2H2 & $1.75 \pm 1.09$ & $0.65 \pm 0.62$ & 2.15 & $3.3 \times 10^{-2}$ \\
\hline ADAMTSL3 & $4.25 \pm 0.95$ & $3.15 \pm 0.84$ & 2.14 & $3.6 \times 10^{-2}$ \\
\hline \multicolumn{5}{|l|}{ Control genes } \\
\hline G3PDH & $4.11 \pm 2.11$ & $4.21 \pm 0.12$ & -1.07 & 0.2273 \\
\hline KRT5 & $10.13 \pm 0.12$ & $10.36 \pm 0.17$ & -1.10 & 0.0155 \\
\hline
\end{tabular}

Data are presented as $\log _{2}$ mean \pm SD unless otherwise stated. ${ }^{\#}$ : involved in tissue development; ${ }^{~}$ : involved in epithelial/epidermal differentiation.

was determined at 4 weeks of culturing at ALI. Interestingly, mRNA expression of HOXA1 and HOXB2 in basal cells showed strong correlation with polarisation of cells (TER), a necessary step for differentiation, but not with differentiation markers MUC5AC or FOXJ1 (figure 3a-f). ELF5 and KRTDAP on the other hand showed some correlation with TER, MUC5AC and FOXJ1 (supplementary figure 1a and b). VGLL1 did not correlate with either TER or differentiation markers (supplementary figure 1c). Compared to normal cells, COPD cells also showed lower expression of occludin and E-cadherin, representatives of tight and adherence junction proteins, respectively (figure $4 \mathrm{a}-\mathrm{c}$ ). These observations indicate that HOXA1 and HOXB2 may participate in polarisation of the cells, which is altered in COPD.

\section{Kinetics of HOXA1 and HOXB2 expression in regenerating airway epithelium}

To determine whether HOXA1 and HOXB2 expression changes during the regeneration of airway epithelium, we assessed the expression of these two genes in basal cells and the confluent basal cells cultured at the ALI for 1, 2 and 4 weeks. In normal cells, while the expression of HOXA1 remained the same throughout the culturing period (figure 5), the expression of HOXB2 increased with time of 

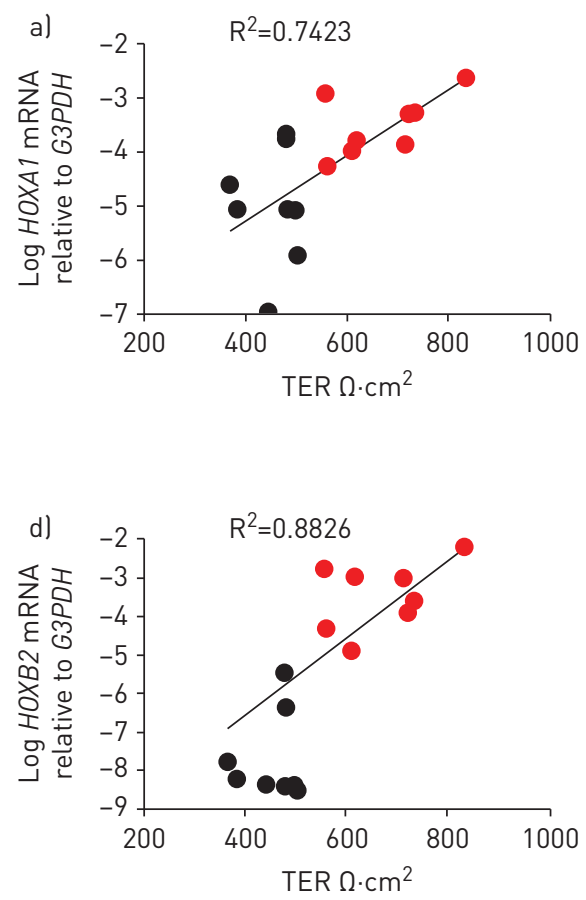
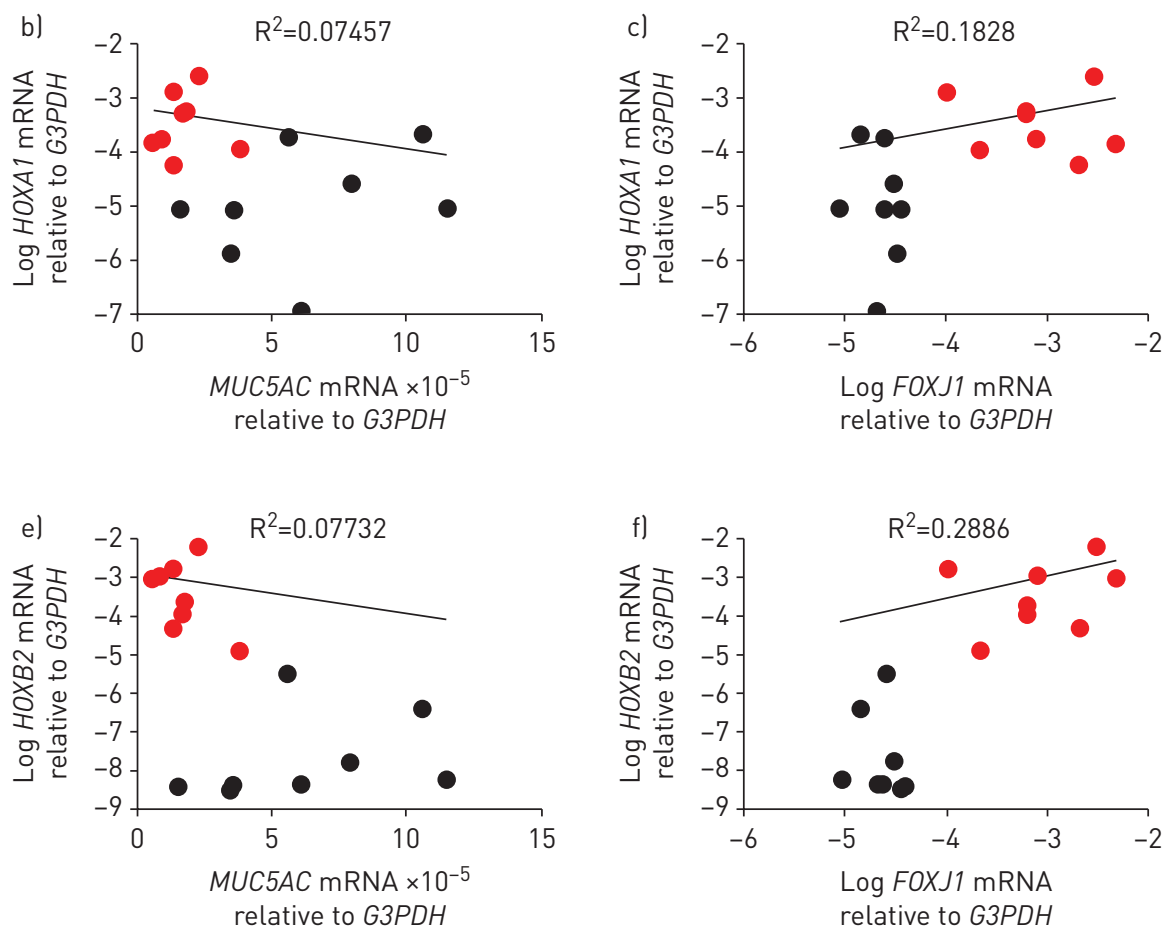

FIGURE 3 Expression of HOXA1 and HOXB2 in basal cells correlates with transepithelial resistance (TER) and not differentiation markers. Basal cells from 8 normal and 8 COPD subjects were cultured at air-liquid interface (ALI) for 2 weeks (to assess TER) or 4 weeks (to assess MUC5AC and FOXJ1). Total RNA was isolated from basal cells and cells cultured at ALI for 4 weeks. mRNA expression of HOXA1 and HOXB2 in basal cells, and MUC5AC and FOXJ1 were determined by probe-based qPCR. The data were normalised to G3PDH. TER measured in cells cultured at ALI for 2 weeks correlates with the expression of HOXA1 and HOXB2 (a and d). The expression of either HOXA1 or HOXB2 does not show strong correlation with differentiation markers MUC5AC or FOXJ1 (b, c, e and f). Red and black dots in all the panels represent data from normal and COPD cells, respectively.

culturing, showing maximum expression at 2 weeks. In COPD cells, expression of both HOXA1 and HOXB2 showed an increasing trend with time of culturing, which did not reach statistical significance. Expression of both genes was significantly lower in COPD than normal cells at all time points.

Because the HOXB2 expression dynamically changed and reached maximum at 2 weeks when the cells polarise, we examined the contribution of HOXB2 in polarisation of cells. We used a bronchial epithelial cell line, 16HBE14o-, which polarises when cultured on a semipermeable membrane. We knocked down HOXB2 in $16 \mathrm{HBE} 14 \mathrm{o}-$ cells by using gene-specific siRNA and assessed cell polarisation by measuring TER, and the expression and localisation of occludin and E-cadherin. Compared to nontargeting (NT) siRNA, HOXB2 siRNA-transfected cells showed significantly lower TER (figure 6a) and this was associated
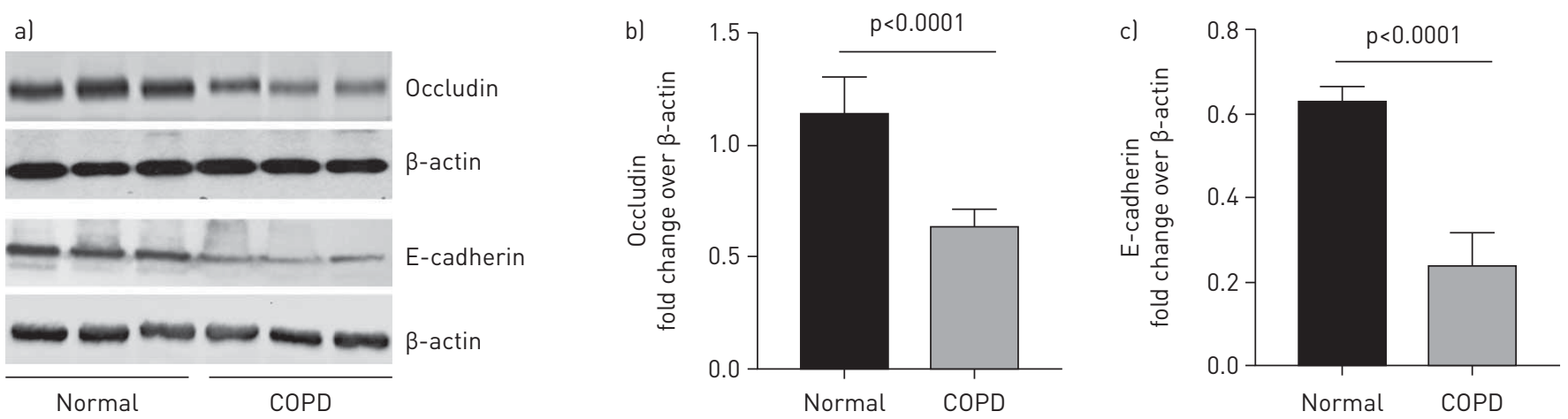

FIGURE 4 COPD cells show reduced expression of E-cadherin and occludin proteins. a) Total protein isolated from polarised cells was subjected to Western blot analysis to determine the protein expression of E-cadherin and occludin. Image is representative of 8 normal and 8 COPD subjects. The density of bands were determined and expressed as fold change over $\beta$-actin ( $b$ and $c$ ). The data represent mean $\pm S D$ calculated from 8 normal and 8 COPD basal cells from 3 independent experiments and the significance was assessed by unpaired t-test. 

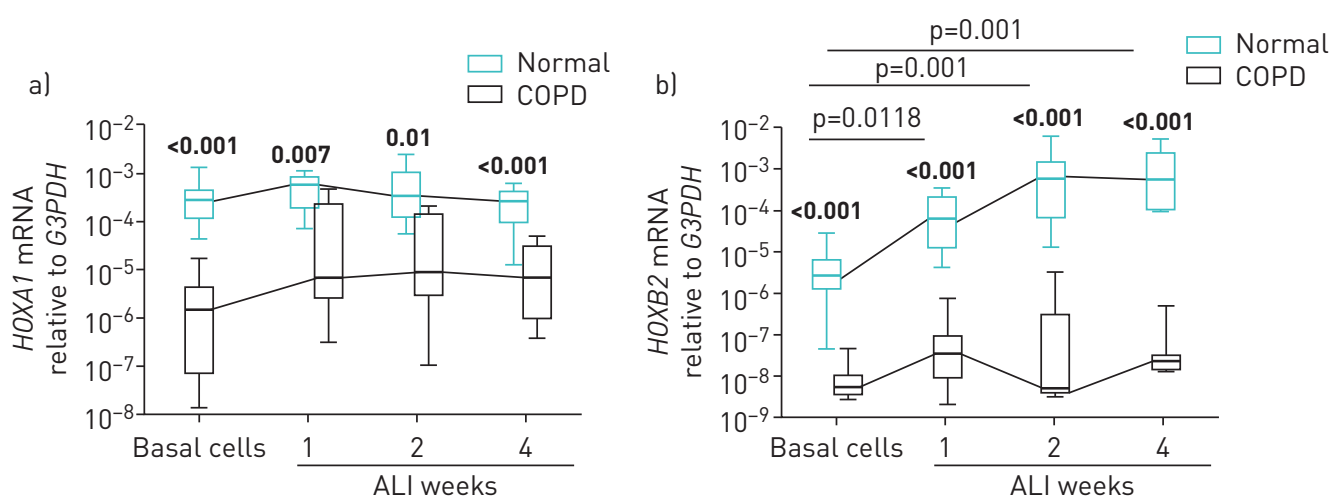

FIGURE 5 HOXB2 expression shows dynamic changes during culturing. Total RNA from basal cells and basal cells cultured at air-liquid interface (ALI) for 1, 2, and 4 weeks was isolated, cDNA was synthesised and subjected to probe-based qPCR to determine the expression of a) HOXA1 and b) HOXB2. The data represent median with range. Statistical significance was determined by ANOVA on ranks with Kruskal-Wallace post hoc test. Numbers in bold represent difference in the expression of genes between normal and COPD cells at each time point. HOXB2 expression significantly changed with time of culturing but not in COPD cells.

with attenuated expression of E-cadherin and occludin in the apicolateral junctions of the cells (figure $6 \mathrm{~b}$ and c). Western blot analysis indicated reduced expression of both occludin and E-cadherin (figure 6d and f). Successful knockdown of HOXB2 was confirmed by Western blot analysis (figure 6g).

\section{Bronchial epithelium in COPD lungs show reduced expression of HOXB2}

Immunofluorescence microscopy was performed to examine the expression of HOXB2 in the second and third branching of bronchi in normal and COPD lung sections. The HOXB2 expression was observed in the bronchial epithelium of both normal and COPD lungs (figure $7 \mathrm{a}$ and $\mathrm{b}$ ), but it was substantially lower in COPD than in normal samples. Expression of HOXB2 was primarily observed in the nuclei of all the cells in the bronchial epithelium. Isotype IgG control showed no signals, indicating the specificity of the HOXB2 antibody (figure 7c).

Taken together, these results indicate that COPD and normal basal cells show significant differences in the expression of certain genes that are involved in tissue development and epithelial/epidermal differentiation. Our studies also indicate that HOXB2 may contribute to polarisation of bronchial epithelial cells.

\section{Discussion}

Airway epithelium from COPD shows an abnormal phenotype and this may be due to altered gene expression in basal cells, which play a fundamental role in regenerating the epithelium [13, 14]. To identify altered regenerative mechanisms and pathways in airway basal cells in COPD, we determined gene expression levels in COPD and normal basal cells by using microarrays. We then confirmed the contribution of one of the developmental genes, HOXB2 in polarisation of cells, which is a necessary step during epithelial regeneration. We also demonstrate that HOXB2 is not only reduced in COPD cells in vitro, but also in the bronchial epithelium of COPD patients.

Comparing transcriptomic profiles of basal cells from COPD and normal subjects, we identified a COPD-specific signature. Genes associated with tissue development and epithelial cell differentiation were underrepresented in COPD basal cells, indicating that regenerating programmes are altered in these cells. Out of the four genes identified under the tissue development category, HOXA1 and HOXB2 are expressed in adult human lungs and the expression is reduced in emphysematous lungs [26], but their role in regeneration of airway epithelium has never been studied. The present study demonstrates that HOXB2 expression is substantially reduced in the bronchial epithelium of COPD patients compared to normal subjects. Furthermore, HOXB2 expression dynamically changes reaching maximum at the time of cell polarisation during the regeneration of normal epithelium in vitro indicating that HOXB2 may contribute to cell polarisation. Consistent with this notion, knockdown of HOXB2 led to reduction in TER and this was associated with reduced expression of E-cadherin and occludin. Further studies are needed to evaluate the mechanisms underlying the regulation of adherence and tight junction proteins by HOXB2.

Microarray analysis also identified reduced expression of DSG1 and actin-binding LIM (ABLIM)3 in COPD cells. DSG1, a major component of intercellular desmosome junctions is involved in the interaction of plaque proteins and intermediate filaments mediating cell-cell adhesion. ABLIM3 interacts with actin 


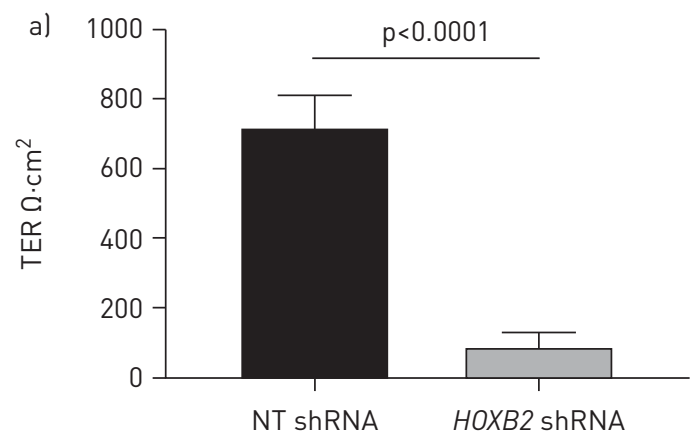

c)

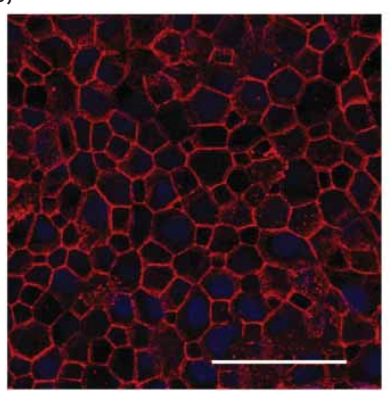

NT shRNA

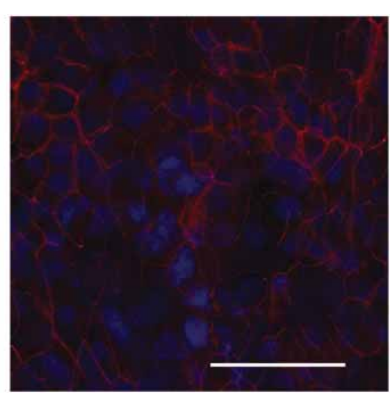

HOXB2 ShRNA b)

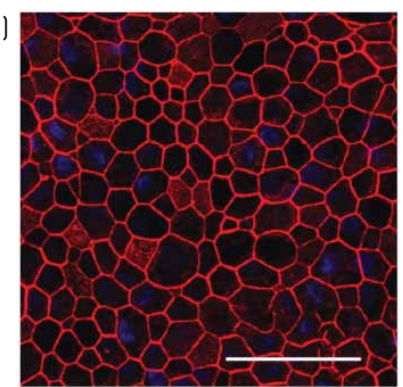

NT shRNA

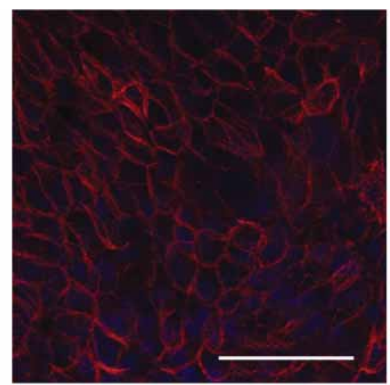

HOXB2 shRNA

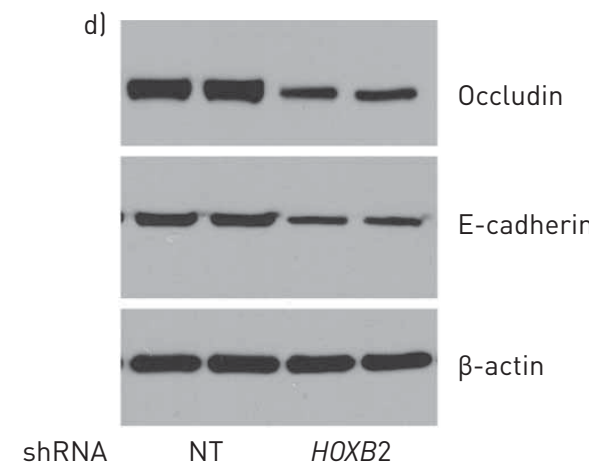

g)
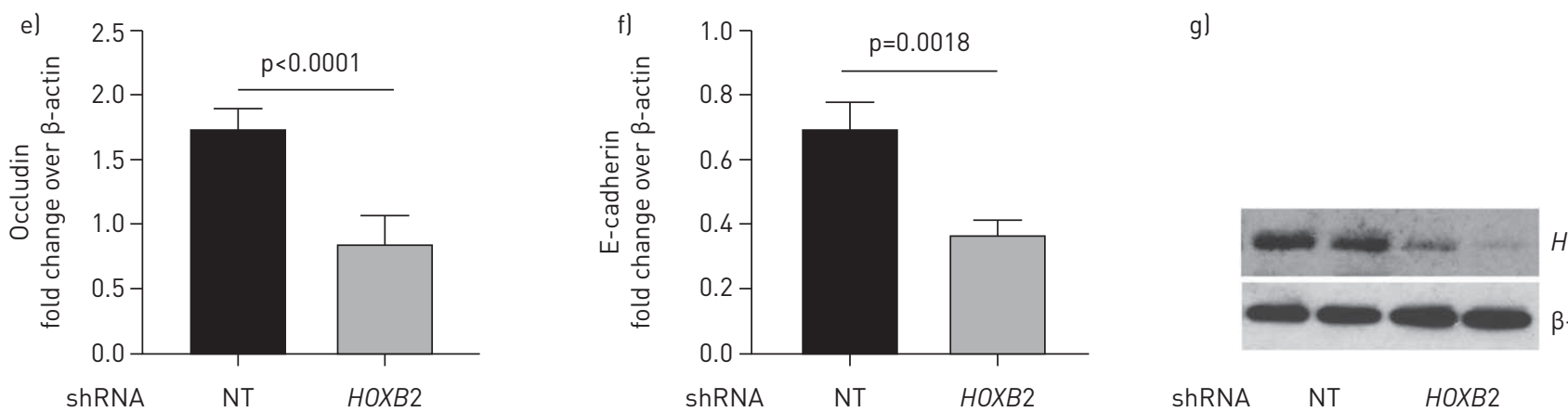

HOXB2

-actin

shRNA NT

HOXB2

FIGURE 6 HOXB2 contributes to epithelial cell polarisation. a) The 16HBE140- cells cultured in Transwells $(90 \%$ confluent) were transfected with nontargeting (NT) or HOXB2 short hairpin (sh)RNA and incubated for another 2 days to promote polarisation and then transepithelial resistance (TER) was measured. Cells were fixed to assess the localisation of b) occludin and c) E-cadherin by immunofluorescence microscopy. d) From identical cultures, total protein was isolated and expression of occludin and E-cadherin was determined by Western blot analysis. Density of the bands was determined and expressed as fold change over $\beta$-actin ( $e$ and f). The data represent mean \pm SD calculated from 4 independent experiments and unpaired t-test was used to calculate the p-value. g) HOXB2 knockdown was confirmed by Western blot analysis. Images in (b, $c$, $\mathrm{d}$ and $\mathrm{g}$ ) are representative of 4 independent experiments. Scale bars $=20 \mu \mathrm{m}$.

filaments and is thought to be a component of adherens junctions [27]. The role of HOXB2 in regulating these genes is yet to be determined and will be addressed in a future study.

The cell-differentiation-associated DEGs included genes encoding cytokeratins (KRT4, 6C and 24), expressed during differentiation of epithelial cells and responsible for their structural integrity; PADI13, involved in the late stages of epidermal differentiation, and ELF5, a member of an epithelium-specific subclass of the Ets transcription factor family that is required for proper lung development and differentiation of epithelial cells [28]. In the present study, ELF5 showed correlation with differentiation markers, MUC5AC and FOXJ1, indicating that ELF5 may contribute bronchial epithelial differentiation. Interestingly, ELF5 maps to 11p13, a genomic region that has been associated with cystic fibrosis lung disease severity in genome-wide association studies $[29,30]$ and can be considered as a COPD risk locus. 
a)
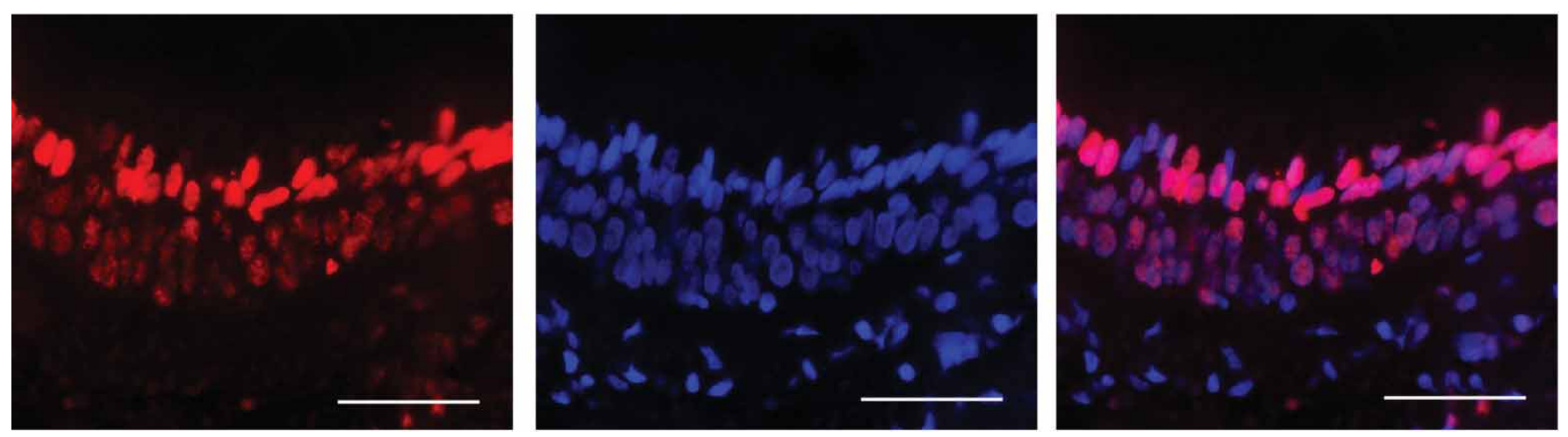

b)
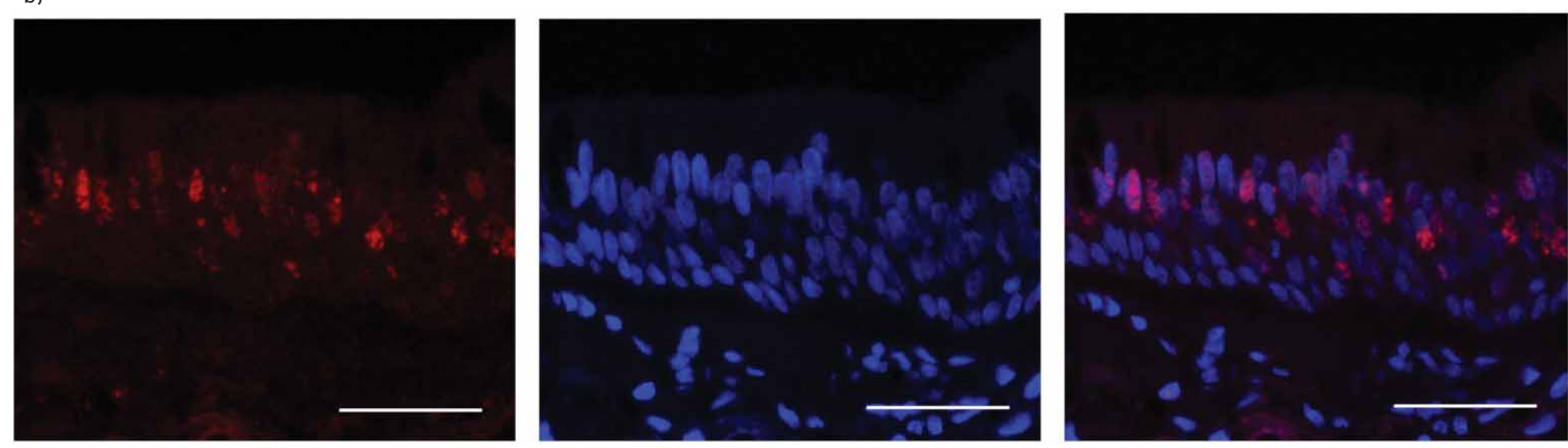

c)

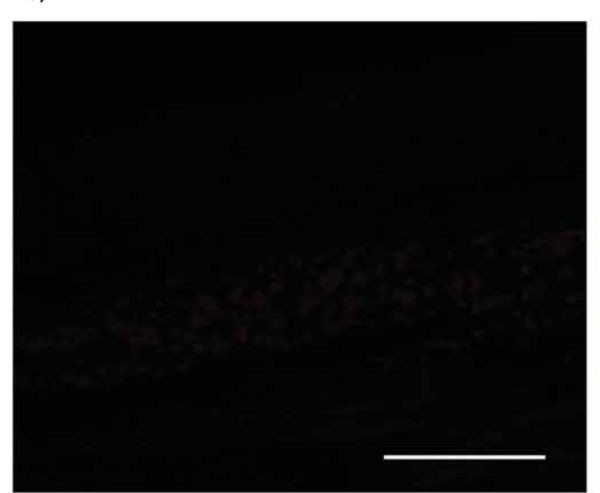

HOXB2

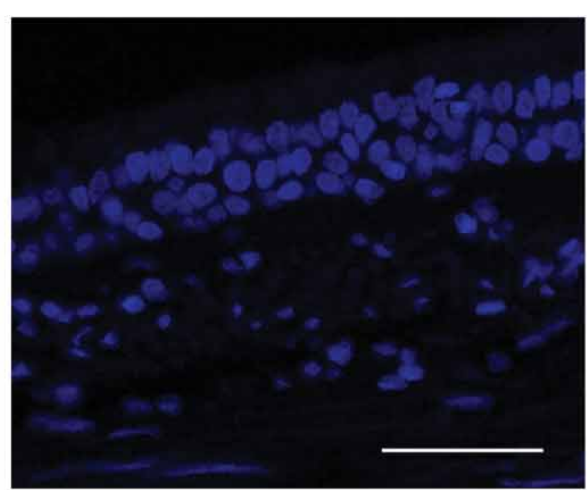

Nuclei

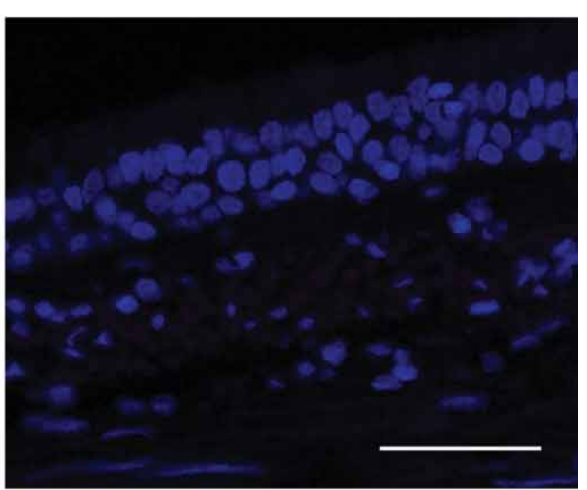

Merged

FIGURE 7 Immunolocalisation of HOXB2 in lung tissues. Paraffin sections of COPD and normal lungs were deparaffinised, incubated for 10 min in boiling $10 \mathrm{mM}$ citrate buffer ( $\mathrm{pH}$ 6.0), allowed to cool down to room temperature. The sections were then treated with $3 \%$ hydrogen peroxide to quench endogenous peroxidase activity, blocked with 5\% normal horse serum containing $0.3 \%$ Triton X-100 for $1 \mathrm{~h}$. Slides were washed and incubated with primary antibody for $16 \mathrm{~h}$ at $4^{\circ} \mathrm{C}$. The slides were washed, incubated with tyramide conjugated with Alexa Fluor 488 and then counterstained with 4',6-diamidino-2-phenylindole. Red, HOXB2 in the left panels, blue, nuclei in the centre panels and magenta, colocalisation of HOXB2 with nuclei in right panels. a) Normal and b) COPD lung section respectively stained with HOXB2 antibody. c) Normal lung section stained with isotype control IgG. The images are representative of lung sections from 6 normal and 6 COPD subjects. Scale bars $=20 \mu \mathrm{m}$.

Interestingly, three other differentially expressed transcripts in our study mapped to COPD risk loci: CNFN in 19q13.2, SNORD111 in 16q22.1, and LOC105374160 in 3q25.1 [15].

DEGs between COPD and normal basal cells also included genes associated with antimicrobial response, all downregulated in COPD: the peptidoglycan recognition proteins 3 and 4 (PGLYRP3 and PGLYRP4) that bind to peptidoglycans of Gram-positive and/or Gram-negative bacteria; the CXCL14 cytokine that plays chemotactic activity for monocytes and may be involved in the homeostasis of monocyte-derived 
macrophages; and the interleukin (IL)-36 receptor antagonist (IL-36RN), a member of the IL-1 cytokine family that specifically inhibits the activation of NF- $\kappa B$ induced by IL-1F6 [31]. The glutathione-specific $\gamma$-glutamyl cyclotransferase 1 (CHAC1), expression of which was upregulated in COPD basal cells, mediates the ATF4-ATF3-DDIT3/CHOP cascade and acts as a pro-apoptotic component of the unfolded protein response. It also regulates the oxidative balance by catalysing the cleavage of glutathione. These results indicate that COPD basal cells may acquire epigenomic changes, which affects expression of genes that are involved in regeneration and maintenance of homeostasis of airway epithelium.

Previously, RNA-sequencing analysis of basal cells from never-smokers and active smokers identified 676 smoking-dysregulated genes [15], but there was no overlap between these genes and the dysregulated genes that were identified in the present study. These observations indicate that the dysregulated genes identified in this study may be specific to end-stage COPD disease, because all the basal cells were obtained during lung transplantation. Moreover, our patients had stopped smoking at least for 6 months prior to surgery, therefore we may have lost the changes in gene expression that occur during active smoking. It would be interesting to compare the transcriptomic profiles generated from former-smoker COPD patients with the ones of active-smoker COPD patients. This requires prospective collection of the tissue that we plan to conduct in the near future.

In summary, basal cells from COPD patients show downregulation of genes associated with epithelial cell differentiation, cell junctions and dysregulation of focal adhesion pathways, all of which may contribute to regeneration of abnormal airway epithelium. $\mathrm{HOXB2}$, one of the downregulated genes in COPD basal cells was found to contribute to polarisation of cells. Finally, compared to normal cells, COPD bronchial epithelium showed reduced HOXB2 protein. These studies provide valuable information for future investigations on the contribution of dysregulated genes in epithelial regeneration and immune responses in COPD.

Acknowledgments: We thank Fernando Martinez, Cornell University, Ithaca, NY, USA, and Catherine Meldrum, University of Michigan, Ann Arbor, MI, USA for helping us to obtain tracheobronchial segments from healthy and COPD subjects. We also thank Biobank Facility of the Lung Center, Temple University for providing lung tissue samples for preparing paraffin blocks. We thank the Microarray Core Facility at the University of Michigan for performing microarrays on normal and COPD samples and assistance with preliminary data analysis.

Conflict of interest: None declared.

Support statement: This work was supported by NIH grants AT007620. Funding information for this article has been deposited with the Crossref Funder Registry.

\section{References}

1 Ganesan S, Comstock AT, Sajjan US. Barrier function of airway tract epithelium. Tissue Barriers 2013; 1 : e24997.

2 Beers MF, Morrisey EE. The three R's of lung health and disease: repair, remodeling, and regeneration. $J$ Clin Invest 2011; 121: 2065-2073.

3 Regamey N, Jeffery PK, Alton EW, et al. Airway remodelling and its relationship to inflammation in cystic fibrosis. Thorax 2011; 66: 624-629.

4 Barnes PJ, Shapiro SD, Pauwels RA. Chronic obstructive pulmonary disease: molecular and cellular mechanisms. Eur Respir J 2003; 22: 672-688.

5 Decramer M, Janssens W, Miravitlles M. Chronic obstructive pulmonary disease. Lancet 2012; 379 : $1341-1351$.

6 Staudt MR, Buro-Auriemma LJ, Walters MS, et al. Airway basal stem/progenitor cells have diminished capacity to regenerate airway epithelium in chronic obstructive pulmonary disease. Am J Respir Crit Care Med 2014; 190: 955-958.

7 Schneider D, Ganesan S, Comstock AT, et al. Increased cytokine response of rhinovirus-infected airway epithelial cells in chronic obstructive pulmonary disease. Am J Respir Crit Care Med 2010; 182: 332-340.

8 Jing Y, Gimenes JA, Mishra R, et al. NOTCH3 contributes to rhinovirus-induced goblet cell hyperplasia in COPD airway epithelial cells. Thorax 2019; 74: 18-32.

9 Ghosh M, Miller YE, Nakachi I, et al. Exhaustion of airway basal progenitor cells in early and established chronic obstructive pulmonary disease. Am J Respir Crit Care Med 2018; 197: 885-896.

10 Ganesan S, Unger BL, Comstock AT, et al. Aberrantly activated EGFR contributes to enhanced IL-8 expression in COPD airways epithelial cells via regulation of nuclear FoxO3A. Thorax 2013; 68: 131-141.

11 Milara J, Peiro T, Serrano A, et al. Epithelial to mesenchymal transition is increased in patients with COPD and induced by cigarette smoke. Thorax 2013; 68: 410-420.

12 Randell SH. Airway epithelial stem cells and the pathophysiology of chronic obstructive pulmonary disease. Proc Am Thorac Soc 2006; 3: 718-725.

13 Crystal RG. Airway basal cells. The "smoking gun" of chronic obstructive pulmonary disease. Am J Respir Crit Care Med 2014; 190: 1355-1362.

14 Shaykhiev R, Crystal RG. Basal cell origins of smoking induced airway epithelial disorders. Cell Cycle 2014; 13: 341-342.

15 Ryan DM, Vincent TL, Salit J, et al. Smoking dysregulates the human airway basal cell transcriptome at COPD risk locus 19q13.2. PLoS ONE 2014; 9: e88051.

16 Cho MH, Castaldi PJ, Wan ES, et al. A genome-wide association study of COPD identifies a susceptibility locus on chromosome 19q13. Hum Mol Genet 2012; 21: 947-957. 
Rock JR, Gao X, Xue Y, et al. Notch-dependent differentiation of adult airway basal stem cells. Cell Stem Cell 2011; 8: 639-648.

18 Gomi K, Arbelaez V, Crystal RG, et al. Activation of NOTCH1 or NOTCH3 signaling skews human airway basal cell differentiation toward a secretory pathway. PLoS ONE 2015; 10: e0116507.

19 Wang R, Ahmed J, Wang G, et al. Down-regulation of the canonical Wnt beta-catenin pathway in the airway epithelium of healthy smokers and smokers with COPD. PLoS ONE 2011; 6: e14793.

20 Danahay $\mathrm{H}$, Pessotti AD, Coote J, et al. Notch2 is required for inflammatory cytokine-driven goblet cell metaplasia in the lung. Cell Rep 2015; 10: 239-252.

21 Yang J, Zuo WL, Fukui T, et al. Smoking-dependent distal-to-proximal repatterning of the adult human small airway epithelium. Am J Respir Crit Care Med 2017; 196: 340-352.

22 Ganesan S, Pham D, Jing Y, et al. TLR2 activation limits rhinovirus-stimulated CXCL-10 by attenuating IRAK-1-dependent IL-33 receptor signaling in human bronchial epithelial cells. J Immunol 2016; 197: 2409-2420.

23 Wang J, Vasaikar S, Shi Z, et al. WebGestalt 2017: a more comprehensive, powerful, flexible and interactive gene set enrichment analysis toolkit. Nucleic Acids Res 2017; 45: W130-W137.

24 Unger BL, Ganesan S, Comstock AT, et al. Nod-like receptor X-1 is required for rhinovirus-induced barrier dysfunction in airway epithelial cells. J Virol 2014; 88: 3705-3718.

25 Faris AN, Ganesan S, Chattoraj A, et al. Rhinovirus delays cell repolarization in a model of injured/regenerating human airway epithelium. Am J Respir Cell Mol Biol 2016; 55: 487-499.

26 Golpon HA, Geraci MW, Moore MD, et al. HOX genes in human lung: altered expression in primary pulmonary hypertension and emphysema. Am J Pathol 2001; 158: 955-966.

27 Matsuda M, Yamashita JK, Tsukita S, et al. abLIM3 is a novel component of adherens junctions with actin-binding activity. Eur J Cell Biol 2010; 89: 807-816.

28 Metzger DE, Stahlman MT, Shannon JM. Misexpression of ELF5 disrupts lung branching and inhibits epithelial differentiation. Dev Biol 2008; 320: 149-160.

29 Wright FA, Strug LJ, Doshi VK, et al. Genome-wide association and linkage identify modifier loci of lung disease severity in cystic fibrosis at 11p13 and 20q13.2. Nat Genet 2011; 43: 539-546.

30 Stolzenburg LR, Yang R, Kerschner JL, et al. Regulatory dynamics of 11p13 suggest a role for EHF in modifying CF lung disease severity. Nucleic Acids Res 2017; 45: 8773-8784.

31 Debets R, Timans JC, Homey B, et al. Two novel IL-1 family members, IL-1 delta and IL-1 epsilon, function as an antagonist and agonist of NF-kappa B activation through the orphan IL-1 receptor-related protein 2. J Immunol 2001; 167: 1440-1446. 